\title{
Effect of surface finishing treatments on the color stability of CAD/CAM materials
}

\author{
Funda Ozen ${ }^{1}$, Nermin Demirkol'2*, Ozge Parlar $\mathbf{O z}^{2}$ \\ ${ }^{1}$ Prosthodontics, Private Practice, Denizli, Turkey \\ ${ }^{2}$ Department of Prosthodontics, Faculty of Dentistry, Gaziantep University, Gaziantep, Turkey
}

\begin{abstract}
PURPOSE. The aim of this study was to evaluate the effect of different surface finishing processes on the color stabilities of lithium disilicate glass-ceramics, zirconia-reinforced lithium silicate ceramics, and resin nanoceramics after artificial ageing. MATERIALS AND METHODS. 216 samples were prepared from 3 different CAD/CAM materials (LAVA Ultimate, IPS e.max CAD, VITA Suprinity) with A1 HT color at a size of $14 \times 12 \mathrm{~mm}$ and a thickness of $0.5 \pm 0.05 \mathrm{~mm}$. Color measurements of the samples were performed with a spectrophotometer using color parameters and CIE Lab color system on a gray backing between baseline color and after 5000 cycles of artificial ageing in 4 stages (i.e. the first measurement before the treatment, the second measurement after polishing, the third measurement after cement application, and the fourth measurement after artificial ageing). The results were evaluated using the Variance analysis and Fisher's LSD test. RESULTS. Resin nanoceramics (LU) exhibited higher color change values than zirconia-reinforced lithium silicate (VS) and lithium disilicate (EC) ceramics after artificial ageing. Manual polishing and glazing resulted in similar color change for LU and VS $(P>.05)$. In the EC group, glazing provided statistically different results as compared to the manual polishing and control groups $(P<.05)$. Among the ceramic groups, color change values of the subgroup, which was treated by glazing, of the zirconia-reinforced lithium silicate (VS) and lithium disilicate (EC) samples were below the clinically acceptable level $(\Delta \mathrm{E}<3.5)$. CONCLUSION. The lowest color change for all stages was observed in Vita Suprinity. [J Adv Prosthodont 2020;12:150-6]
\end{abstract}

KEYWORDS: CAD/CAM; Ceramics; Surface finishing processes; Color stability

\section{INTRODUCTION}

Today, digital workflow systems let dentists design and manufacture dental restorations in a single appointment. Restorations produced using computer-aided design and computer-aided manufacturing (CAD/CAM) systems can be designed and milled in one session by a dentist. However,

\footnotetext{
Corresponding author:

Nermin Demirkol

Department of Prosthodontics, Faculty of Dentistry, Gaziantep University, Şahinbey, 27310 Gaziantep, Turkey

Tel. +905064810477: e-mail,dt_nerminhamdemirci@hotmail.com Received November 28, 2019 / Last Revision May 7, 2020 / Accepted May 14,2020

(C) 2020 The Korean Academy of Prosthodontics

This is an Open Access article distributed under the terms of the Creative Commons Attribution Non-Commercial License (http://creativecommons. org/licenses/by-nc/4.0) which permits unrestricted non-commercial use, distribution, and reproduction in any medium, provided the original work is properly cited.
}

This work was supported by Gaziantep University Scientific Research Projects Governing Unit [Project Number: DHF.UT.18.06]. the mentioned milling process does not provide a smooth surface that is completely ready for cementation. ${ }^{1}$ Color stability that can be affected by surface roughness is an important parameter in addition to the esthetic and mechanical properties of ceramic restorations for the success and longevity of the restorations. ${ }^{2}$ Glazing is a method for obtaining a smooth surface. Indirect dentistry required try-in appointments, and the delivery should be performed only when no adjustment was required. However, the glaze layer can be removed by clinical adjustment of the restoration in time and this can result in increased surface roughness. ${ }^{3}$ The long-term natural appearance of restorations with suitable cement space and appropriate surface finishing treatment can be described as an advantageous alternative. ${ }^{4,5}$ Also, surface structure and roughness are effected by surface finishing treatment; although chairside polishing is possible and effective, it should be limited. Surface finishing treatment has a critical effect on the color stability and long-term success of restorations. Disks, polishing kits, and polishing pastes can be applied in accordance with the manufacturers' 
recommendation to obtain smooth surfaces by mechanical polishing. ${ }^{2}$

As a result of the adaptation procedures performed on the patient's restoration during delivery, a rough surface is formed, which necessitates intraoral finishing and polishing to be performed on the restoration. ${ }^{6}$ Polishing and finishing treatments are done to minimize the wear effect on the opposing teeth by reducing the abrasiveness of the restoration and to ensure hygiene by preventing bacteria from adhering to the restoration surface. ${ }^{7}$ Previous studies have shown that finishing treatment increases the restoration's color stability. ${ }^{8,9}$ Even when the color selection is optimal, the material can exhibit clinically recognizable color changes depending on the oral environment. ${ }^{10}$ Various surface finishing treatments can be applied to esthetic CAD/CAM restorations. The most commonly preferred surface finishing treatment before cementation is glazing in a porcelain furnace. Moreover, recent studies have shown that a smooth and bright surface can also be obtained by using manual polishing as well as glazing. ${ }^{1,5,11}$

The aim of this study was to compare the effects of different surface finishing treatments applied to lithium disilicate glass-ceramic, zirconia-reinforced lithium silicate ceramic, and resin nanoceramic, which are routinely used in CAD/CAM systems on the color stability after artificial ageing. The hypotheses of the study are as follows: groups that undergo glazing would have less color change in comparison to the groups that undergo manual polishing, and all groups would exhibit significant color changes as a result of the ageing process.

\section{MATERIALS AND METHODS}

The study tested lithium disilicate glass-ceramic (EC; IPS e.max CAD, Ivoclar Vivadent, AG, Shaan, Lichtenstein, Lot: X39499), zirconia-reinforced lithium silicate ceramic (VS; VITA Suprinity, VITA Zahnfabrik H. Rauter GmbH \& Co. KG, Bad Saeckingen, Germany, Lot: 63323), and polymerized resin nanoceramic (LU; LAVA Ultimate, 3M ESPE, St. Paul, MN, USA, Lot: N945036) CAD/CAM blocks for evaluation the color stability. Variolink N (Ivoclar Vivadent AG, Shaan, Lichtenstein) resin cement in translucent shades was used. Resin cement was applied to all samples after performing two different suitable finishing treatments on three different ceramic materials. Afterward, thermal ageing was applied.
This study design consisted of 24 specimens in each group at $80 \%$ power for statistical differences between groups according to power analysis with $G$ power program 3.1.9.2 version. A total of $216(\mathrm{~N}=216)$ specimens were prepared with $24(\mathrm{n}=24)$ specimens in each subgroup. Ceramic blocks with A1 HT color at a size of $14 \times 12 \times 18$ $\mathrm{mm}$ and a thickness of $0.5 \pm 0.05 \mathrm{~mm}$ were prepared using a precision cutting machine (Isomet 1000, Buehler, Lake Bluff, IL, USA). The samples were cut at low speed under cool water with $0.8 \mathrm{~mm}$ intervals, also considering the thickness $(0.3 \mathrm{~mm})$ of the diamond blade (Buehler, Lake Bluff, IL, USA). Samples were polished with 400-, 600-, and then 800- grit silicon carbide papers (3M ESPE, St. Paul, MN, USA) under running water for 15 seconds to standardize the surface quality. Their thickness was measured using a digital micrometer (Minitech 233 Presi, Grenoble, France) that has $0.01 \mathrm{~mm}$ accuracy and repeatability.

Among the prepared samples, EC and VS groups were crystallized in a Programat P300 furnace (Ivoclar Vivadent AG, Shaan, Lichtenstein) in accordance with the manufacturer's instructions to complete crystallization. This way, 216 ceramic samples with 3 different structures at a size of $14 \times$ $12 \times 0.5 \mathrm{~mm}$ were obtained. Before the measurement of color, the samples were kept in an ultrasonic cleaner (Easyclean, Renfert, IL, USA) using distilled water for 10 minutes and dried. They were kept under room temperature in a dark, dry environment before the spectrophotometric measurements.

The measurements were conducted using daylight illuminant D65 with a natural gray background inside a viewing booth according to the CIE Lab color system as shown in formula 1 above with a spectrophotometer (Vita Easyshade, VITA-Zahnfabrik, Bad Säckingen, Germany). Basic shade measurement mode was selected from tool options for spectrophotometer. As a result of the measurements, L (lightness), a, and b (chromatic values) values of the ceramic samples were obtained in the CIE Lab system. A standard white disc was measured in intervals specified by the manufacturer to calibrate the spectrophotometer.

Following the first color measurements, the samples were assigned to three subgroups using simple randomization for each ceramic group, wherein the first group was treated with manual polishing, the second group was treated with glazing, and the third group was assigned to be the control group. Different polishing methods applied to each group are shown in Table 1 and Table 2. Manual polishing

Table 1. Glazing and polishing materials

\begin{tabular}{cll}
\hline Groups & \multicolumn{1}{c}{ Manual polishing materials } & \multicolumn{1}{c}{ Glaze materials } \\
\hline LU & Sof-Lex Spiral System Kit (3M ESPE) & $\begin{array}{l}\text { Light-cure Optiglaze (GC) (as light-polymerized glazing agent for LU was not } \\
\text { available, Optiglaze; GC applied) }\end{array}$ \\
EC & Optrafine F/P/HP (Ivoclar Vivadent AG) & Ivocolor glaze paste/fluo (Ivoclar Vivadent AG) \\
VS & Vita Suprinity Polishing Set (clinical) & Vita Akzent Plus glaze powder/fluid (VITA-Zahnfabrik) \\
\hline
\end{tabular}


Table 2. The polishing material content

\begin{tabular}{ll}
\hline $\begin{array}{l}\text { Sof-Lex Spiral } \\
\text { Step } 1\end{array}$ & Pystem Kit (3M ESPE) \\
Step 2 & Polishing using 'pink' \\
Kit type & Diamond Polishing System Kit \\
& \\
Optrafine F/P/HP (Ivoclar Vivadent AG) \\
Step 1 & Pre-polishing using 'light blue' \\
Step 2 & Polishing using 'dark blue \\
Step 3 & High-gloss polishing with brushes and polishing \\
Kit type & paste \\
Viamond Polishing System Kit \\
Step 1 & Pre-polishing using 'pink' \\
Step 2 & Polishing using 'grey' \\
Kit type & Diamond Polishing System Kit
\end{tabular}

continued for 30 seconds at $10,000 \mathrm{rpm}$ on a single surface for each sample with finger pressure by the same clinician. Manual polishing discs were replaced after every five samples. The samples were kept in an ultrasonic cleaner for 10 seconds following manual polishing, and then dried. In the glazing process, a layer of a suitable polish was applied to each sample in accordance with the manufacturer's instructions. After the surface finishing process, the second color measurements of the manual polishing, glazing, and control groups were carried out with the same standards. Polishing and glazing materials for studied ceramic types are shown in Table 1 and contents of polishing kits are shown in Table 2.

A roughened surface was obtained by air abrasion in the LU group, and by hydrofluoric acid application for $20 \mathrm{sec}-$ onds in the EC and VS groups. A $0.1 \mathrm{~mm} \pm 0.05 \mathrm{~mm}$-thick index was prepared to apply the resin cement. After roughening, a thin coat of Monobond N (Ivoclar Vivadent, AG, Shaan, Lichtenstein) was applied with a brush to the pretreated surfaces. Material was allowed to react for $60 \mathrm{sec}-$ onds by the manufacturer's instruction. Subsequently, any remaining excess was dispersed with a strong stream of air. $0.1 \mathrm{~mm} \pm 0.05 \mathrm{~mm}$-thick of Variolink $\mathrm{N}$ (Ivoclar Vivadent AG, Shaan, Lichtenstein) translucent resin cement was applied to the ceramic samples in accordance with the manufacturer's instructions and light cured for 20 seconds. The third color measurements were conducted under the same standards previously prepared according to the CIE Lab color system after completion of the polymerization. Color measurements were repeated three times and the mean value was used for each sample.

After the third color measurements, the samples were placed in a thermal ageing device (SD Mechatronik Thermocycler, Feldkirchen-Westerham, Germany) with a 30-second waiting and 10 -second transfer time at $5^{\circ} \mathrm{C}$ and $55^{\circ} \mathrm{C}$ for thermal ageing without any solution. In total, 5,000 cycles were undertaken for each sample. Following the ageing process, the samples were washed with distilled water and dried. Afterwards, the fourth color measurements were conducted with the same standards for each group.

As a result of the spectrophotometric measurements, the first measurements $\left(\mathrm{L} 1 *, \mathrm{a} 1^{*}, \mathrm{~b} 1^{*}\right)$, the second measurements $\left(\mathrm{L} 2^{*}, \mathrm{a} 2^{*}, \mathrm{~b} 2^{*}\right)$ after polishing, the third measurements $\left(\mathrm{L}^{*}, \mathrm{a} 3^{*}, \mathrm{~b} 3^{*}\right)$ after cementation, and the fourth measurements (L4*, a $\left.4^{*}, \mathrm{~b} 4^{*}\right)$ after thermal ageing were used to obtain $\Delta \mathrm{E}$ values using the following formula:

Formula 1.

$$
\Delta \mathrm{E}=\left[(\Delta \mathrm{L})^{2}+(\Delta \mathrm{a})^{2}+(\Delta \mathrm{b})^{2}\right]^{1 \backslash 2}
$$

The analysis was conducted using SPSS 22.0 software. In accordance with the obtained results, mean color change values $(\overline{\mathrm{X}} \Delta \mathrm{E})$ and standard deviations were expressed by groups. Among the multiple comparison tests, Fisher's LSD test was used to assess the differences among the groups. Factorial Analysis of Variance was used for the repeated measurements in data analysis. Among the multiple comparison tests, the LSD test was used to assess the differences among the groups $(P<.05)$.

\section{RESULTS}

The results and statistical analysis of color change $\Delta \mathrm{E}$ values before surface treatment, and after polishing and glaze procedures of LU, EC, and VS groups are presented in Table 3.

Table 3. The statistical analyses of color change $(\Delta \mathrm{E})$ values

\begin{tabular}{|c|c|c|c|c|}
\hline \multirow{2}{*}{$\begin{array}{l}\text { Color } \\
\text { change }\end{array}$} & \multirow{2}{*}{ Groups } & \multicolumn{3}{|c|}{$P$ values } \\
\hline & & Between M-G & Between M-C & Between G-C \\
\hline \multirow[t]{3}{*}{$\Delta \mathrm{E} 1 \_2$} & LU & .9356 & .0409 & .0494 \\
\hline & EC & $.0008^{\star}$ & $0^{*}$ & $0^{*}$ \\
\hline & VS & .3053 & $.0004^{*}$ & $0^{*}$ \\
\hline \multirow[t]{3}{*}{$\Delta \mathrm{E} 2 \_3$} & LU & $.0048^{*}$ & .0138 & $0^{*}$ \\
\hline & EC & $0^{*}$ & $.0012^{*}$ & .2534 \\
\hline & VS & .7051 & .5522 & .3308 \\
\hline \multirow[t]{3}{*}{$\Delta$ E3_4 } & LU & .3454 & $.0014^{*}$ & $0^{*}$ \\
\hline & EC & .0999 & .0119 & .3811 \\
\hline & VS & .9747 & .8258 & .8012 \\
\hline \multirow[t]{3}{*}{$\Delta \mathrm{E} 1 \_4$} & LU & .7756 & .3292 & .2077 \\
\hline & EC & $0^{*}$ & $.0003^{\star}$ & $.0001^{*}$ \\
\hline & VS & .5873 & .6923 & .3483 \\
\hline
\end{tabular}

$P<.005, \Delta \mathrm{E}=$ color change

$\triangle E 1 \_2$ = color change of 1. and 2. measurement (base color-after polishing), $\triangle \mathrm{E} 2 \_3=$ color change of 2 . and 3. measurement (after polishing-after cementation), $\triangle \mathrm{E} 3 \_4=$ color change of 3 . and 4 . measurement (after cementation-after artificial ageing), $\Delta \mathrm{E} 1 \_4$ = color change of 1 . and 4 . measurement (base color-after artificial ageing), $\mathrm{M}=$ manual polishing, $\mathrm{G}=$ glaze, $\mathrm{C}=$ control 
In LU group, statistically significant difference was found between the first color measurement (pure block) and the second color measurement after manual polishing was obtained. The mean color change was $\overline{\mathrm{X}} \Delta \mathrm{E}$ manual $=1.14$ and $\overline{\mathrm{X}} \Delta \mathrm{E}$ glaze $=1.09$ after the manual polishing and glazing treatments, respectively. According to these values, a statistically significant difference was not observed between the manual polishing and glazing treatments in terms of color change $(P=.935)$. Manual polishing group exhibited statistically significant results in comparison to the control group $(P=$ .040). Glazing group exhibited statistically significant results in comparison to the control group $(P=.049)$.

In LU group that underwent manual polishing, the color change after cement application was $\left(\Delta \mathrm{E} 2 \_3\right) \overline{\mathrm{X}} \Delta \mathrm{E}$ manual $=$ 3.68 , whereas the color change in the glazing and control groups was $\overline{\mathrm{X}} \Delta \mathrm{E}$ glaze $=2.09$ and $\overline{\mathrm{X}} \Delta \mathrm{E}$ control $=5.06$, respectively. Statistically significant differences were found between the groups: manual polishing and glazing groups in terms of color change after cement application $(P=.004)$, manual polishing and control groups $(P=.013)$, glazing and control groups $(P=.000)$, respectively.

The color change $\left(\Delta \mathrm{E} 3 \_4\right)$ between the completion of cement application and completion of the thermal cycle was $\overline{\mathrm{X}}_{\Delta \mathrm{E} \text { manual }}=5.08, \overline{\mathrm{X}}_{\Delta \mathrm{E} \text { glaze }}=5.61$, and $\overline{\mathrm{X}}_{\Delta \mathrm{E} \text { control }}=$ 3.29 for the manual polishing, glazing, and control groups, respectively. A statistically significant difference was not observed between the manual polishing and glazing groups in terms of the color change after thermal cycling $(P=$ .345). Statistically significant differences between the manual polishing and control groups $(P=.001)$ and also between the glazing and control groups $(P=.000)$ were found in LU group.

In the group $L U$, the color change ( $\left.\Delta \mathrm{E} 1 \_4\right)$ from the first color measurement until the final color measurement after the completion of the surface finishing treatment, cementation and thermal cycle was $\overline{\mathrm{X}} \Delta \mathrm{E}$ manual $=5.10, \overline{\mathrm{X}} \Delta \mathrm{E}$ glaze $=$ 4.94, and $\overline{\mathrm{X}} \Delta \mathrm{E}$ control $=5.64$ in the manual polishing, glazing and control groups, respectively. Statistically significant difference was not found between the following groups: manual polishing and glazing groups in terms of color change between the first and final color measurements $(P=.775)$, manual polishing group and the control group that did not undergo polishing $(P=.329)$, glazing and control groups $(P$ $=.207$ ).

There was a statistically significant relationship in group $\mathrm{EC}$, between $\Delta \mathrm{E}^{*}$ and finishing $(P<.05)$. The mean color change was $\overline{\mathrm{X}} \Delta \mathrm{E}$ manual $=2.12$ and $\overline{\mathrm{X}} \Delta \mathrm{E}$ glaze $=3.50$ for the manual polishing and glazing groups, respectively. There was a statistically significant difference between the $\Delta \mathrm{E} 1 \_2$ values after manual polishing and glazing $(P=.000)$. The color change after glazing and manual polishing was statistically significant as compared to the control group $(P=$ $.000)$.

In EC group that underwent manual polishing, the color change $\left(\Delta \mathrm{E} 2 \_3\right)$ after cement application was $\overline{\mathrm{X}} \Delta \mathrm{E}$ manual $=$ 3.14 , whereas the color change in the glazing and control groups was $\overline{\mathrm{X}} \Delta \mathrm{E}$ glaze $=1.34$ and $\overline{\mathrm{X}} \Delta \mathrm{E}$ control $=1.81$, respec- tively. Statistically significant difference was obtained between the following groups: manual polishing and glazing groups in terms of color change after cement application $(P$ $=.000)$, manual polishing and control groups $(P=.001)$, glazing and control groups in terms of color change after cement application $(P=.253)$.

The mean $\Delta \mathrm{E}$ values following thermal cycle was $\overline{\mathrm{X}} \Delta \mathrm{E}$ manual $=5.72, \overline{\mathrm{X}} \Delta \mathrm{E}$ glaze $=5.05$, and $\overline{\mathrm{X}} \Delta \mathrm{E}$ control $=4.69$, for the manual polishing, glazing, and control groups, respectively. There was no statistically significant difference between the manual polishing and glazing groups in terms of the $\Delta \mathrm{E} 3 \_4$ values after the thermal cycle $(P=.099)$. Statistically significant differences were not found between the manual polishing and control groups $(P=.011)$ and between the glazing and control groups $(P=.381)$ for EC group.

The color change ( $\left.\Delta \mathrm{E} 1 \_4\right)$ from the first color measurement until the final color measurement after the completion of polishing, cementation, and thermal cycle was $\overline{\mathrm{X}} \Delta \mathrm{E}$ manual $=5.72, \bar{X}_{\Delta \mathrm{E} \text { glaze }}=2.64$, and $\overline{\mathrm{X}} \Delta \mathrm{E}$ control $=4.24$ in the manual polishing, glazing, and control groups, respectively. In comparison between the first and fourth color measurements, a statistically significant difference was achieved between manual polishing and glazing groups $(P=.000)$. Statistically significant differences were found between the following groups: the manual polishing group and the control group that did not undergo polishing $(P=.000)$, and also glazing and control groups $(P=.000)$.

In VS group, there was a statistically significant difference between the first color measurement (pure block) and the second color measurement after manual polishing $(P<.05)$. The mean color change was $\overline{\mathrm{X}} \Delta \mathrm{E}$ manual $=1.53$ and $\overline{\mathrm{X}} \Delta \mathrm{E}$ glaze $=1.97$ after the manual polishing and glazing treatments, respectively. According to these values, a statistically significant difference was not observed between the manual polishing and glazing treatments, in terms of color change $(P=$ .305). Manual polishing group exhibited statistically significant results in comparison to the control group $(P=.000)$. Glazing group exhibited statistically significant results in comparison to the control group $(P=.000)$.

In VS group that applied manual polishing, the color change $\left(\Delta \mathrm{E} 2 \_3\right)$ after cement application was $\overline{\mathrm{X}} \Delta \mathrm{E}$ manual $=$ 1.88 , whereas the color change in the glazing and control groups was $\overline{\mathrm{X}} \Delta \mathrm{E}$ glaze $=2.04$ and $\overline{\mathrm{X}} \Delta \mathrm{E}$ control $=1.62$, respectively. A statistically significant difference was not observed between the manual polishing and glazing groups in terms of the color change after cement application $(P=.705)$. Statistically significant differences were not found between the following groups; manual polishing and control groups $(P=.552)$ also glazing and control groups $(P=.330)$.

The color change $\left(\Delta \mathrm{E} 3 \_4\right)$ between the completion of cement application and completion of the thermal cycle was $\overline{\mathrm{X}} \Delta \mathrm{E}$ manual $=3.18, \overline{\mathrm{X}} \Delta \mathrm{E}$ glaze $=3.16$, and $\overline{\mathrm{X}} \Delta \mathrm{E}$ control $=$ 3.27 for the manual polishing, glazing, and control groups, respectively. A statistically significant difference was not observed between the manual polishing and glazing groups in terms of the color change after thermal cycle $(P=.974)$. 
Statistically significant difference was not obtained between manual polishing-control groups $(P=.825)$ and also glazing-control groups $(P=.801)$ for VS group.

In VS group, the color change ( $\left.\Delta \mathrm{E} 1 \_4\right)$ from the first color measurement until the final color measurement after the completion of the surface finishing treatment, cementation, and thermal cycle was $\overline{\mathrm{X}} \Delta \mathrm{E}$ manual $=2.65, \overline{\mathrm{X}} \Delta \mathrm{E}$ glaze $=$ 2.42 , and $\overline{\mathrm{X}} \Delta \mathrm{E}$ control $=2.83$ in the manual polishing, glazing, and control groups, respectively. There was no statistically significant difference between manual polishing and glazing groups in terms of color change between the first and the final color measurements $(P=.587)$. Statistically significant difference was not achieved between the manual polishing group-the control group that did not undergo polishing $(P$ $=.692)$ and also glazing group-the control group that did not undergo polishing $(P=.348)$.

\section{DISCUSSION}

Hypothesis of the study are as follows: groups that undergo glazing would have less color change in comparison to the groups that undergo manual polishing, and all groups would exhibit significant color changes as a result of the ageing process. Glazing in a group of EC ceramics significantly affected the color change, and therefore the hypothesis was accepted for EC ceramics. On the other hand, glazing did not have less color change in comparison to the groups that undergo manual polishing in the group of LU and VS ceramics, and therefore the hypothesis was rejected for LU and VS ceramics. Based on these findings, initial hypothesis of the study has been partially rejected.

In the present study, three different CAD/CAM restorative materials with a resin cement were applied with different polishing treatments and artificial ageing in order to investigate color change. In literature, various CAD/CAM blocks (IPS emax CAD, IPS emax Press, IPS emax Ceram, Vita Suprinity, Vita Enamic, Vita Mark II and Cerec blocks) have been studied frequently. ${ }^{2,5,12-14}$ Aesthetic, smooth and bright surfaces can be achieved with various polishing and final procedures in CAD/CAM restorations. Optiglaze, Vita Akzent Plus, and IPS Ivocolor Glaze systems are the most preferred glazing procedures. Additionally, Meisinger Polishing Set, Top Glaze, Silco-pol, Renfert Polishing LiSi2, Vita Suprinity Polishing Kit systems have been used for manual polishing procedure in the literature.,5 Optiglaze, IPS Ivocolor Glaze, Vita Akzent Plus Glaze were used for glazing, and Sof-lex spiral system kit, Optrafine and Vita Suprinity Polishing Set were used for manual polishing procedure in this study.

Turgut and Bagis ${ }^{15}$ showed that the light cure and dual cure resin cements affected the porcelain laminate veneer ceramics after UV ageing corresponding to 1 year of clinical service. Mina et al. ${ }^{16}$ showed that resin cements affected the final color of restoration after 30 days of ageing using different resin cements but did not show a long term difference. In the current study, thermocycling was performed with 5000 cycles corresponding to 6 months of ageing simi- lar to that used in the study by Alp et al. ${ }^{12}$ The results of present study showed that the significant color changes achieved in all groups after cementation may be related to the water absorption and surface properties (glazing or polishing) of these materials.

In all samples, LU samples exhibited the highest color change values, whereas VS samples had lower values in comparison to the other groups. As a result of the accelerated artificial ageing of the specimens, all groups showed significant color changes following cementation. Regarding surface treatments, the aforementioned different finishing treatments did not result in a statistically significant difference in the LU and VS groups. However, in the EC group, glazing provided a statistically different difference as compared to the manual polishing and control groups.

It has been reported that porcelain thickness, repeated firings, surface finishing treatments, and surface roughness can affect the color stability of porcelain restorations. ${ }^{8}$ Many investigators have reported that different polishing techniques for ceramic restorations can be an alternative to glazing procedure. ${ }^{8,17}$ It is very difficult to recognize minor color changes in dental materials with human eye. Previous studies reported that values lower than 1.1 cannot be perceptible with the human eye, whereas values higher than 3.3 are clinically unacceptable. ${ }^{18,19}$ Perceptibility means the finding of color difference between a tooth and a near colored restoration, and acceptability means having the color difference that would be acceptable for that restoration. ${ }^{12}$ According to perceptibility and acceptability thresholds, manually polished LU and VS with all surface treatments showed lower color changes than the clinically perceptible level. Also, glazed and manual polished EC showed lower color changes than the clinically acceptable level in this study.

Kilinc and Turgut ${ }^{5}$ reported that Lava Ultimate had the highest color change compared to the other ceramics (Vita Enamic, Vita Suprinity and Vita Mark II) after different finishing treatment. Alp et al. ${ }^{12}$ showed that color changes in IPS e.max CAD and Vita Suprinity after applying different surface treatments were clinically acceptable, similar to the present study. Kanat-Erturk ${ }^{2}$ found that glazing had lower color change than mechanical polishing and external staining- glazing for IPS e.max CAD and Vita Suprinity ceramics, supported by the results of the current study for EC and VS. Palla $e t$ al. ${ }^{13}$ reported that IPS e.max CAD and IPS e.max Press non-glaze had the lowest $\mathrm{E}$ value after the different thermal ageing process compared to other CAD/ CAM ceramics (IPS e.max Press non-glaze and IPS e.max Ceram). In our study, although the color change of the manual polishing group in the LU group was lower than the glazing group, the last color change after the thermal cycle affected the manual varnish and glaze group in a similar way and gave statistically different results from the control groups. For LU ceramics, glazing did not have less color change in comparison to the manual polishing group. Therefore, the hypothesis was rejected for LU ceramics. Color change remained above the clinically acceptable value in all subgroups of the LU group. For the EC group, color 
change after the thermal cycle affected the manual varnish, glaze, and control group in a similar way. Color difference results revealed that glazing in a group of EC ceramics significantly affected the color differences. Therefore, the hypothesis was accepted for EC ceramics. For the VS group, color change after thermal cycle affected the manual polish, glaze, and control group statistically similarly. Glazing did not have less color change in comparison to the manual polishing groups in VS ceramics. Therefore, the hypothesis was rejected for VS ceramics.

It was reported by the manufacturer that in Vita Suprinity ceramic material, zirconia particles were distributed homogenously with a finer molecular structure within the glass structure, thereby enhancing material strength and ensuring a more homogenous surface structure. Moreover, it aimed to combine the translucent and esthetic properties of lithium silicate with the strength of zirconia in this ceramic material (ZLS). ${ }^{20}$ After crystallization, surface structure of ZLS was studied using a scanning electron microscope. The surface of ZLS material has a homogenous, fine, and rodlike crystalline structure with an average crystal size of approximately $0.5 \mathrm{~mm} \cdot{ }^{18,21,22}$ Such lower color change values for VS observed in this study can be attributed to the homogenous structure of VS with finer crystal particles containing zirconia.

Karaokutan $e t$ al. ${ }^{14}$ compared the color stability of LU, IPS e.max CAD, and feldspathic glass ceramic after applying 300 hours of artificial ageing in their study. They reported that the color change value of Lava Ultimate resin nanoceramics was significantly higher than the color change values of the others. Lava Ultimate resin nanoceramic materials contain $80 \%$ zirconia and silica nanoparticles by weight and they are reinforced with polymer matrix that contains crosslinks. ${ }^{23}$ The main reason for the color change in resincontaining composite materials was reported to be the water absorption level due to the hydrophillic/hydrophobic properties of the resin matrix. ${ }^{24}$ Resin nanoceramic structure contains Bis-GMA. Hydroxyl groups within Bis-GMA increase viscosity and they are not stable in terms of color change. TEGDMA and UDMA were added to the structure in order to reduce viscosity and enhance mechanical properties by increasing crosslinks. However, color stability is still questionable. In addition, most of the polymers contain functional groups in molecular chains that absorb UV light. It was reported that in the artificial ageing process, the composite structure absorbs water and disrupts the filler and resin structure, leading to the rupture of the chemical bonds. ${ }^{25}$ According to the study by Gawriołek et al. ${ }^{26}$ ceramic materials exhibit better color stability in comparison to the composite materials. In our study, Vita Suprinity group showed the highest color stability. In our study, the highest change value between the first and last color measurements was observed in the LU group. The lowest color stability of LU can be explained by the chemical structure of LU resin nanoceramics according to result of this study.

It cannot be concluded that the thermal ageing process employed in this study is the same as the clinical conditions.
This is because materials are only processed depending on the temperature variables throughout the thermal ageing process. Moreover, it is considerably challenging to evaluate the response of dental materials to color change in the long term with clinical studies. The artificial ageing process carried out in a laboratory environment can be considered as an important method for researchers to standardize the studies on dental material characteristics. It helps the researcher save time by detecting the amount of material degradation in the thermal cycle within a shorter period of time, while the researcher gains information about the material. ${ }^{27-30}$

In this in vitro study, three different $\mathrm{CAD} / \mathrm{CAM}$ material samples were compared in terms of color stability. The last stage, i.e. artificial ageing, may not be enough to assess the clinical performance of full-ceramic materials. The extent to which color differences affect the clinical performance of full-ceramics should be evaluated in clinical studies. The limitations of this study are that it was an in vitro study and the material was stained on both surfaces. In the clinical situation, the ceramic is bonded to tooth structure and exposed to different types of liquids. Additionally, only one thickness was evaluated in this study. In future studies, the effects of cement shade, dentin color, and thicknesses of materials should be investigated on the color stability.

\section{CONCLUSION}

In terms of color stability findings, glazing had statistically different effect on the color change between the first color measurement and the final color measurement compare to manual polishing on EC group $(P=.000)$. Therefore, glazing can be recommended for EC ceramics. For the LU and VS groups, the color change between the first color measurement and the final color measurement after the completion of the surface finishing treatment, cementation, and thermal cycle was similar in the manual polishing, glazing, and control groups, respectively. Significant differences were not found among the groups $(P>$.005). Therefore, manual polishing can be recommended as an alternative to glazing for LU and VS ceramics.

\section{ORCID}

Funda Ozen bttps://orcid.org/0000-0001-7701-6156

Nermin Demirkol https://orcid.org/0000-0002-2415-5977

Ozge Parlar Oz bttps://orcid.org/0000-0002-8927-3448

\section{REFERENCES}

1. Fasbinder DJ, Neiva GF. Surface evaluation of polishing techniques for new resilient $\mathrm{CAD} / \mathrm{CAM}$ restorative materials. J Esthet Restor Dent 2016;28:56-66.

2. Kanat-Ertürk B. Color stability of CAD/CAM ceramics prepared with different surface finishing procedures. J Prosthodont 2020;29:166-72.

3. Akar GC, Pekkan G, Çal E, Eskitaşçığlu G, Özcan M. Effects of surface-finishing protocols on the roughness, color 
change, and translucency of different ceramic systems. J Prosthet Dent 2014;112:314-21.

4. Barizon KT, Bergeron C, Vargas MA, Qian F, Cobb DS, Gratton DG, Geraldeli S. Ceramic materials for porcelain veneers: part II. Effect of material, shade, and thickness on translucency. J Prosthet Dent 2014;112:864-70.

5. Kilinc H, Turgut S. Optical behaviors of esthetic CAD-CAM restorations after different surface finishing and polishing procedures and UV aging: An in vitro study. J Prosthet Dent 2018;120:107-13.

6. Amaya-Pajares SP, Ritter AV, Vera Resendiz C, Henson BR, Culp L, Donovan TE. Effect of finishing and polishing on the surface roughness of four ceramic materials after occlusal adjustment. J Esthet Restor Dent 2016;28:382-96.

7. Anusavice KJ, Kakar K, Ferree N. Which mechanical and physical testing methods are relevant for predicting the clinical performance of ceramic-based dental prostheses? Clin Oral Implants Res 2007;18:218-31.

8. Motro PF, Kursoglu P, Kazazoglu E. Effects of different surface treatments on stainability of ceramics. J Prosthet Dent 2012;108:231-7.

9. Vichi A, Fabian Fonzar R, Goracci C, Carrabba M, Ferrari M. Effect of finishing and polishing on roughness and gloss of lithium disilicate and lithium silicate zirconia reinforced glass ceramic for CAD/CAM systems. Oper Dent 2018;43:90-100.

10. Dos Santos DM, da Silva EVF, Watanabe D, Bitencourt SB, Guiotti AM, Goiato MC. Effect of different acidic solutions on the optical behavior of lithium disilicate ceramics. J Prosthet Dent 2017;118:430-6.

11. Lawson NC, Burgess JO. Gloss and stain resistance of ceramic-polymer CAD/CAM restorative blocks. J Esthet Restor Dent 2016;28:S40-5.

12. Alp G, Subasi MG, Johnston WM, Yilmaz B. Effect of surface treatments and coffee thermocycling on the color and translucency of CAD-CAM monolithic glass-ceramic. J Prosthet Dent 2018;120:263-8.

13. Palla ES, Kontonasaki E, Kantiranis N, Papadopoulou L, Zorba T, Paraskevopoulos KM, Koidis P. Color stability of lithium disilicate ceramics after aging and immersion in common beverages. J Prosthet Dent 2018;119:632-42.

14. Karaokutan I, Yilmaz Savas T, Aykent F, Ozdere E. Color stability of CAD/CAM fabricated inlays after accelerated artificial aging. J Prosthodont 2016;25:472-7.

15. Turgut S, Bagis B. Colour stability of laminate veneers: an in vitro study. J Dent 2011;39:e57-64.

16. Mina NR, Baba NZ, Al-Harbi FA, Elgezawi MF, Daou M. The influence of simulated aging on the color stability of composite resin cements. J Prosthet Dent 2019;121:306-10.

17. Alghazali N, Hakami AA, Alajlan GA, Alotaibi RM, Alabdulwahab FN, AlQuraishi LA, Abdalkadeer H, Al Moaleem MM. Influence of the arabic-coffee on the overall color of glazed or polished porcelain veneers - In vitro study. Open Dent J 2019;13:364-70.

18. Ardu S, Braut V, Gutemberg D, Krejci I, Dietschi D, Feilzer AJ. A long-term laboratory test on staining susceptibility of esthetic composite resin materials. Quintessence Int 2010;41:695-702.

19. Kuehni RC, Marcus RT. An experiment in visual scaling of small color differences. Color Res App 1979;4:83-91.

20. Preis V, Behr M, Hahnel S, Rosentritt M. Influence of cementation on in vitro performance, marginal adaptation and fracture resistance of CAD/CAM-fabricated ZLS molar crowns. Dent Mater 2015;31:1363-9.

21. Belli R, Wendler M, de Ligny D, Cicconi MR, Petschelt A, Peterlik H, Lohbauer U. Chairside CAD/CAM materials. Part 1: Measurement of elastic constants and microstructural characterization. Dent Mater 2017;33:84-98.

22. Ramos Nde C, Campos TM, Paz IS, Machado JP, Bottino MA, Cesar PF, Melo RM. Microstructure characterization and SCG of newly engineered dental ceramics. Dent Mater 2016; 32:870-8.

23. Acar O, Yilmaz B, Altintas SH, Chandrasekaran I, Johnston WM. Color stainability of CAD/CAM and nanocomposite resin materials. J Prosthet Dent 2016;115:71-5.

24. Rocca GT, Bonnafous F, Rizcalla N, Krejci I. A technique to improve the esthetic aspects of CAD/CAM composite resin restorations. J Prosthet Dent 2010;104:273-5.

25. Ferracane JL. Hygroscopic and hydrolytic effects in dental polymer networks. Dent Mater 2006;22:211-22.

26. Gawriołek M, Sikorska E, Ferreira LF, Costa AI, Khmelinskii I, Krawczyk A, Sikorski M, Koczorowski PR. Color and luminescence stability of selected dental materials in vitro. J Prosthodont 2012;21:112-22.

27. Hamza TA, Alameldin AA, Elkouedi AY, Wee AG. Effect of artificial accelerated aging on surface roughness and color stability of different ceramic restorations. Stomatol Dis Sci 2017;1:8-13.

28. Dos Santos PH, Catelan A, Albuquerque Guedes AP, Umeda Suzuki TY, de Lima Godas AG, Fraga Briso AL, BedranRusso AK. Effect of thermocycling on roughness of nanofill, microfill and microhybrid composites. Acta Odontol Scand 2015;73:176-81.

29. Subaş1 MG, Alp G, Johnston WM, Yilmaz B. Effects of fabrication and shading technique on the color and translucency of new-generation translucent zirconia after coffee thermocycling. J Prosthet Dent 2018;120:603-8.

30. Yuan JC, Barão VAR, Wee AG, Alfaro MF, Afshari FS, Sukotjo C. Effect of brushing and thermocycling on the shade and surface roughness of CAD-CAM ceramic restorations. J Prosthet Dent 2018;119:1000-6. 